\title{
LIMK1/2 inhibitor LIMKi 3 suppresses porcine oocyte maturation
}

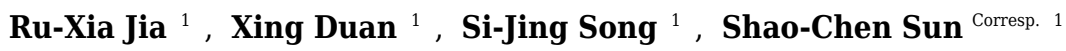 \\ ${ }^{1}$ College of Animal Science \& Technology, Nanjing Agricultural University, Nanjing, China \\ Corresponding Author: Shao-Chen Sun \\ Email address: sunsc@njau.edu.cn
}

LIMKi 3 is a specific selective LIMK inhibitor against LIMK1 and LIMK2, while LIMK1 and LIMK2 are the main regulators of actin cytoskeleton to participate in many cell activities. However, the effect of LIMKi 3 in porcine oocyte meiosis is still unclear. The present study was designed to investigate the effects of LIMKi 3 and potential regulatory role of LIMK1/2 on porcine oocyte meiotic maturation. Immunofluorescent staining of $p$-LIMK1/2 antibody showed that LIMK1/2 was localized mainly to the cortex of porcine oocyte, which colocalized with actin. After LIMKi 3 treatment, the diffusion of COCs became weak and the rate of polar body extrusion was decreased. This could be rescued by moving oocytes to fresh medium. After prolonging the culture time of oocytes, the maturation rate of porcine oocyte increased in LIMKi 3 groups, indicating that LIMKi 3 may suppress the cell cycle during porcine oocyte maturation. We also found that after LIMKi 3 treatment actin distribution was significantly disturbed at porcine oocyte membranes and cytoplasm, indicating the conserved roles of LIMK1/2 on actin dynamics. Next we examined the meiotic spindle positioning in porcine oocyte, and the results showed that a majority of spindles were not attached to the cortex of porcine oocyte, indicating that LIMKi 3 may affect actin-mediated spindle positioning. Taken together, these results showed that LIMK1/2 inhibitor LIMKi 3 had a repressive role on porcine oocyte meiotic maturation. 
2 Ru-Xia Jia, Xing Duan, Si-Jing Song, Shao-Chen Sun*

3

4 College of Animal Sciences and Technology, Nanjing Agricultural University, Nanjing, China.

5

6 Running Title: LIMK1/2 on porcine oocyte

7

8 "Correspondence to: Shao-Chen Sun, College of Animal Science and Technology, Nanjing

9 Agricultural University, Nanjing, China. Tel/Fax: +86-25-84399092. E-mail: sunsc@njau.edu.cn 10 


\section{Abstract}

LIMKi 3 is a specific selective LIMK inhibitor against LIMK1 and LIMK2, while LIMK1 and LIMK2 are the main regulators of actin cytoskeleton to participate in many cell activities. However, the effect of LIMKi 3 in porcine oocyte meiosis is still unclear. The present study was designed to investigate the effects of LIMKi 3 and potential regulatory role of LIMK $1 / 2$ on porcine oocyte meiotic maturation. Immunofluorescent staining of $\mathrm{p}$-LIMK1/2 antibody showed that LIMK1/2 was localized mainly to the cortex of porcine oocyte, which co-localized with actin. After LIMKi 3 treatment, the diffusion of COCs became weak and the rate of polar body extrusion was decreased. This could be rescued by moving oocytes to fresh medium. After prolonging the culture time of oocytes, the maturation rate of porcine oocyte increased in LIMKi 3 groups, indicating that LIMKi 3 may suppress the cell cycle during porcine oocyte maturation. We also found that after LIMKi 3 treatment actin distribution was significantly disturbed at porcine oocyte membranes and cytoplasm, indicating the conserved roles of LIMK1/2 on actin dynamics. Next we examined the meiotic spindle positioning in porcine oocyte, and the results showed that a majority of spindles were not attached to the cortex of porcine oocyte, indicating that LIMKi 3 may affect actin-mediated spindle positioning. Taken together, these results showed that LIMK1/2 inhibitor LIMKi 3 had a repressive role on porcine oocyte meiotic maturation. 
The meiosis in mammalian oocyte is arrested at diplotene stage of first meiotic prophase,

45

manifested by the germinal vesicle (GV) located at the center of the oocyte. Meiotic resumption from diplotene stage is characterized by germinal vesicle breakdown (GVBD). Chromatins condense into chromosomes and microtubules then are organized into the specialized barrelshaped bipolar spindle. When all the chromosomes line up on the equatorial plate, oocyte enters metaphase I. Subsequently, the spindle relocates from the center of the oocyte to a position near the cortex in an actin filament-dependent manner (Almonacid et al. 2014). In addition, actin is enriched to form an actin cap over the spindle, and cortical granules (CGs) become redistributed to form a CG-free domain (CGFD) where microvilli are lost (Deng et al. 2003). And these events are regarded as cortical reorganization. After cortical reorganization, the contractile ring forms and then facilitates cytokinesis of the oocyte to generate a highly polarized egg with the emission of first polar body (Liu 2012; Maddox et al. 2012). Oocyte maturation is important for fertilization and following embryo development (Maro \& Verlhac 2002). Spindle and actin filaments are essential for the extrusion of polar body during the mammalian oocyte maturation (Sun \& Kim 2013).

59 LIM kinase (LIMK) which includes LIMK1 and LIMK2, belongs to serine kinases and is discovered more than a decade ago, and LIMK is shown to be a regulator of actin dynamics (Stanyon \& Bernard 1999). LIM kinase is distinguished by unique structural features: a protein kinase region at the C-terminal, a PDZ-like motif at the interposing region, and two tandemly arrayed LIM domains at the N-terminaland (Ohashi et al. 1994; Okano et al. 1995). Previous studies showed that LIMK regulated the actin polymerization mediated by the Rho family, 
including Rho (Maekawa et al. 1999), Rac (Yang et al. 1998), and Cdc42 (Sumi et al. 1999).

Meanwhile, activated LIMK regulated actin reorganization via phosphorylation and inactivation of cofilin, a family of actin-binding proteins directly involved in the depolymerization and nucleation of actin filaments (F-actin) (Arber et al. 1998; Bamburg 1999). The functions of LIMK contributes to its irreplaceable effects in cell movement, division and structure formation (Bernard 2007). More extensively, LIMK actively promoted pathologic cancer cell division where drastic changes had taken place in actin filaments. HeLa cell as a model cancer cells has been used to investigate the roles of LIMK, and results showed that LIMK-mediated cofilin phosphorylation was required for precise spindle positioning to ensure the achievement of division during mitosis (Kaji et al. 2008). The regulation of LIMK is not only involved in mitosis but also meiosis. In Xenopus oocyte, XLIMK-cofilin system was required for the organization of the microtubulederived precursor of the meiotic spindle (Takahashi et al. 2001). Additionally, LIMK activity was essential for microtubule organizing center organization and distribution in mouse oocyte meiosis (Li et al. 2016). However, little is known regarding the study related with LIMK in porcine oocyte. LIM kinase Inhibitor I, LIMKi 3, is a cell-permeable, potent inhibitor of LIM kinase (IC50 = 7 and $8 \mathrm{nM}$ against LIMK1 and LIMK2, respectively) and shown to effectively destabilize F-actin structure in MDA-MB-231 breast cancer cells (3 to $10 \mu \mathrm{M})$ with concomitant blockage of invasion (by $93 \%$ at $10 \mu \mathrm{M}$ ). Here, we selected porcine as experiment animal to investigate the possible consequence of LIMKi 3 in oocyte meiosis. The treatment of LIMKi 3 significantly affected porcine oocyte maturation. We also showed that LIMKi 3 treatment caused aberrant actin distribution and spindle positioning, which might have contributed to a failure of porcine oocyte 
86

87

88

89

90

91

92

maturation.

\section{Results}

\section{P-LIMK1/2 expression in porcine oocyte}

The subcellular localizations of p-LIMK-1/2 at different stages of meiosis were assessed by immunofluorescent staining. As shown in Figure 1A, p-LIMK-1/2 was primarily localized at cortex of oocyte during all meiotic stages, and this localization is similar with actin. Oocytes of GV stage were obtained and were cultured for 26 and $44 \mathrm{~h}$, which corresponded to the times to achieve the MI and MII stages, respectively. Using western blot, we found that p-LIMK-1/2 protein was all expressed at the MI and MII stages (Fig. 1B).

\section{LIMKi 3 treatment suppresses polar body extrusion in vitro}

Based on the examination of LIMK1/2 expression and localization in porcine oocytes, we next examined whether LIMK1/2 inhibitor LIMKi 3 affected porcine oocyte maturation. As shown in Figure 2A, COCs exhibited different diffusion situation between control and treatment group: the diffusion of $200 \mu \mathrm{M}$ LIMKi 3 treated-COCs was significantly weaker than the control group. In the standard of COCs surrounded by the cumulus cell layers, we counted the proportion of diffusion and found that the rate of good diffusion was decreased significantly $(40.05 \pm 3.03 \%$ vs. $20.50 \pm 4.05 \% ; \mathrm{p}<0.05)$. Oocytes were then obtained from COCs after hyaluronidase treatment, we found that few oocytes extruded the polar body after $200 \mu \mathrm{M}$ LIMKi 3 treatment. In addition, the rate of polar body extrusion of oocytes was reduced in a dose-dependent manner (Fig. 2B). For controls, the ratio of polar body extrusion was $73.44 \pm 5.18 \%(n=123)$; while oocytes were treated 
107

108

109

110

111

112

113

114

115

116

117

118

119

120

121

122

123

124

125

126

127

with LIMKi 3 in the concentration of $50 \mu \mathrm{M}, 100 \mu \mathrm{M}, 150 \mu \mathrm{M}, 200 \mu \mathrm{M}$, the ratio decreased to

$65.66 \pm 3.24 \%(n=95 ; p>0.05), 58.04 \pm 5.58 \%(n=111 ; p<0.05), 54.76 \pm 3.00 \%(n=101 ; p<0.05)$,

$46.88 \pm 5.04 \%(n=135 ; \mathrm{p}<0.01)$. Therefore, the porcine oocyte maturation was suppressed in the

treatment of LIMKi 3, and $200 \mu \mathrm{M}$ was chosen as the appropriate concentration for subsequent

experiments.

To furthur clarifly the effects of LIMKi 3 on porcine oocyte maturation, we prolonged the culture time of porcine oocyte to 60 hours. As shown in figure $2 \mathrm{C}$, after prolonging the culture time, the maturation rate increased compared with $44 \mathrm{~h}$ culture group $(70.68 \% \pm 6.99 \%$ vs. $47.61 \%$ $\pm 7.92 \%)$. Thus, LIMKi 3 repressed the cell cycle of porcine oocyte maturation; To further confirm the inhibitory effects of LIMKi 3, we did the rescue experiment. Compared with oocytes before rescue $(78.18 \% \pm 4.69 \%$ vs. $47.61 \% \pm 7.92 \%$; $<<0.01)$, LIMKi 3 treated oocytes reached the MII stage $(200 \mu \mathrm{M})$ after an additional $16 \mathrm{~h}$ of culture in fresh medium, with the ratio of $73.06 \% \pm$ 3.09\% (figure 2D). Therefore, LIMKi 3 may have a repressive effect on porcine oocyte maturation and its effect was reversible.

\section{LIMKi 3 treatment causes disruption of actin distribution in porcine oocyte}

Considering that LIMK1/2 has been known to regulate actin cytoskeleton, we next examined the effects of LIMK inhibitor LIMKi 3 on actin filament distribution of meiosis I in porcine oocyte. As shown in Figure 3A (10X), compared with the control oocytes, actin fluorescence intensity of LIMKi 3 treated oocytes became much weaker. Furthermore, actin distribution was disturbed both at the membrane and cytoplasm of oocyte after LIMKi 3 treatment (Fig. 3A [40X]). The value of actin fluorescence intensity in porcine oocyte was analyzed by Image J software (Fig. 3B). For 
128 129 130

membrane actin fluorescence intensity, the rate of LIMKi 3 treated oocytes was significantly lower than that in control oocytes $(78.88 \pm 16.53$ vs. $48.18 \pm 9.14 ; \mathrm{P}<0.01)$. Compared with control oocytes, the rate of cytoplasmic actin also decreased after LIMKi 3 treatment $(29.76 \pm 9.02$ vs. $22.70 \pm 7.81 ; \mathrm{P}<0.01)$. These results indicated that the LIMKi 3 treatment resulted in an abnormal actin distribution.

\section{LIMKi 3 treatment causes abnormity of spindle positioning in porcine oocyte}

Spindle migration in meiosis I is actin dependent, so we next investigated the effects of LIMK1/2 inhibitor LIMKi 3 on spindle positioning at MI stage. As shown in Figure 4A (10X), most of the spindles exhibited at the position distant with the cortex after treatment with $200 \mu \mathrm{M}$ LIMKi 3, which is different with that the spindles of control oocytes that exhibited peripheral localization. Enlarged images more clearly showed the differences between control and treatment (Fig. 4A $[40 \mathrm{X}])$.

We also analyzed the cell cycle stage after culture for 26 hours. As shown in Figure 4B, the percentage of oocytes with spindles closed to cytoplasm center at the MI stage for LIMKi 3 treatment was clearly higher than that of control oocytes $(57.11 \pm 7.85 \%$, $\mathrm{n}=212$ vs. $26.58 \pm 5.18 \%$, $\mathrm{n}=234)(\mathrm{p}<0.01)$, nevertheless the percentage of oocytes with spindles at the cortex in the MI stage (35.27 $\pm 7.94 \%$ vs. $59.16 \pm 11.19 \% ; \mathrm{p}<0.01)$ and in the anaphase/telophase (AT) stage $(3.27 \pm 4.53 \%$ vs. $9.61 \pm 6.04 \%$; $<<0.05$ ) were reduced after LIMKi 3 treatment. Meanwhile, the percentage of oocytes at the GVBD stage showed no significant difference between control and treatment $(4.65 \pm 3.08 \%$ vs. $4.35 \pm 4.00 \%$; $>0.05)$. These results demonstrated that LIMKi 3 treatment effectively affected spindle positioning. 


\section{Discussion}

In present study, we investigated the potential roles of LIMK1/2 inhibitor LIMKi 3 during

porcine oocyte maturation. The results showed that LIMKi 3 treatment inhibited the diffusion of

COCs and the emission of polar body, which affected porcine oocyte maturation. Moreover, the

inhibition of LIMKi 3 disrupted actin distribution and meiotic spindle positioning. We speculated

that LIMK1/2 inhibitor LIMKi 3 suppressed porcine oocyte maturation through its effects on actin-

based spindle positioning.

We first examined LIMK1/2 expression in porcine oocyte, and we found that LIMK1/2

existed at the cortex of oocyte in porcine during meiosis, which is similar with actin filament. We

next examined whether LIMK1/2 inhibitor LIMKi 3 affected porcine oocyte maturation. After

LIMKi 3 treatment, the diffusion of COCs became weak, together with the decreased rate of polar

body extrusion, these result indicated that LIMKi 3 suppressed porcine oocyte maturation. In

COCs, there is a mutual promoting relationship between oocyte and granulosa cells. Oocyte is

crucial in the granulosa differentiation and development (Gilchrist et al. 2008). Likewise,

granulosa cells also play regulating roles in the nutrition and signal transduction for oocyte

development and maturation (Sutton-McDowall et al. 2010). Therefore, it is hard to examine

whether the suppression of oocyte maturation by LIMKi 3 is through the effects of LIMKi 3 on

cumulus expansion. Similarly, LIMK has been found to involve oocyte maturation in Xenopus

168 (Takahashi et al. 2001). Upon microinjection of Xenopus-Limk (Xlimk1/2), abundant LIMK

significantly inhibited the appearance of a white maturation spot (WMS), an indicator of entry into 
170

171

172

173

174

175

176

177

178

179

180

181

182

183

184

185

186

187

188

189

190

Xenopus oocyte meiosis, which indicated the failure of Xenopus oocyte maturation. Additionally, the activators of LIMK, which include Rho (Zhang et al. 2014a), Rac (Halet \& Carroll 2007) and Cdc42 (Dehapiot et al. 2013; Ma et al. 2006), are also required for polar body extrusion during the meiotic maturation of mammalian oocyte. Therefore, our results showed that LIMK1/2 inhibitor LIMKi 3 could suppress the polar body extrusion during porcine oocyte maturation.

LIMK catalyzes phosphorylation of an N-terminal $3^{\text {rd }}$ serine residue of cofilin and inhibits its activity to depolymerize actin, thereby leading to stabilization of actin (Arber et al. 1998; Yang et al. 1998). Our results showed similar localization pattern of LIMK $1 / 2$ and actin filaments. We then investigated whether LIMK1/2 participated in the regulation of actin filament during porcine oocyte meiotic maturation. Staining with phalloidin-TRITC we found that LIMKi 3 treatment affected actin distribution in the membrane and cytoplasm of porcine oocyte showing with the decreased rate of actin fluorescence intensity. It has been reported that actin flow droves oocytes progressed to MII stage, which is characterized by asymmetric polar body emission of oocyte (Yi \& Li 2012). Thus, the results demonstrated that LIMK also regulated the actin distribution, which furthur disrupted polar body extrusion in porcine oocyte meiosis. Similar results were observed with Rho-kinase (ROCK), the upstream protein of LIMK1/2, which was shown to be responsible for actin filament distribution which contributed to asymmetric division in meiotic oocyte (Duan et al. 2014; Zhang et al. 2014b). Therefore, our results indicated that the effects of LIMK1/2 inhibitor LIMKi 3 to porcine oocyte maturation may be triggered by its effects on actin distribution.

Actin drives meiotic spindle migration and anchor to ensure spindle positioning accurately, 
191

192

193

194

195

196

197

198

199

200

201

202

203

204

205

206

207

208

209

210

211

which is required for the achievement of oocyte nuclear maturation (Almonacid et al. 2014; Brunet \& Verlhac 2011). Here, we next examined the positioning of spindle after LIMKi 3 treatment. The results showed that most spindles of inhibitor-treated oocytes primarily localized at the position distant with the cortex after $26 \mathrm{~h}$ in culture, the time point by which the meiotic spindles of most oocytes should have attached to the cortex or even entered anaphase I. The results indicated the failure of spindle movement after LIMKi 3 treatment during porcine oocyte meiotic maturation.

Our results were similar with the study on bovine which showed the ROCK/LIMK/cofilin pathway to meiotic spindle migration during oocyte maturation. Therefore, LIMK1/2 inhibitor LIMKi 3 may affect spindle positioning via regulation of actin dynamic during oocyte meiotic maturation in porcine.

In conclusion, our results demonstrated that LIMK1/2 inhibitor LIMKi 3 treatment disrupted actin microfilaments distribution and caused the failure of spindle positioning, which affected porcine oocyte maturation.

\section{Materials and Methods}

\section{Antibodies and chemicals}

Basic maturation culture medium was TCM 199 (Sigma, St. Louis, MO, USA). A rabbit polyclonal p-LIMK-1/2 antibody was from Santa Cruz (Santa Cruz, CA, USA). An Alexa Fluor 488 secondary antibody was from Invitrogen (Carlsbad, CA, USA). Phalloidin-TRITC and mouse monoclonal anti- $\alpha$-tubulin-FITC antibody were from Sigma. LIMK inhibitor LIMKi 3 was from Calbiochem (Darmstadt, Germany). 
212

213

214

215

216

217

218

219

220

221

222

223

224

225

226

227

228

229

230

231

232

\section{Oocyte collection and culture}

Animal use was conducted in accordance with the Animal Research Institute Committee guidelines of Nanjing Agricultural University, China. This study was specifically approved by the Committee of Animal Research Institute, Nanjing Agricultural University, China. Porcine ovaries were collected from a local slaughterhouse, and were then transported to laboratory within 3 hours in sterile saline $(0.9 \% \mathrm{NaCl})$ containing $500 \mathrm{IU} / \mathrm{mL}$ of penicillin and $500 \mathrm{IU} / \mathrm{mL}$ of streptomycin at $37^{\circ} \mathrm{C}$. Cumulus-oocyte complexes (COCs) were obtained from medium-sized follicles of ovaries by aspirating with a 20-gauge needle attached to a 5-ml disposable syringe. Oocytes surrounded by a compact cumulus mass and also with uniform ooplasm were selected and transferred to Dulbecco's PBS, then washed three times with modified medium 199 containing

0.1\% (wt/vol) polyvinyl alcohol, $0.91 \mathrm{mM}$ sodium pyruvate, $3.05 \mathrm{mM}$ glucose, $75 \mathrm{mg} / \mathrm{L}$ of penicillin, and $50 \mathrm{mg} / \mathrm{L}$ of streptomycin. Only oocytes with intact cumulus cells and evenly granulated ooplasm were chosen for in vitro maturation (IVM).

A group of 80 COCs were transferred to $500 \mu \mathrm{L}$ of maturation medium consisting of $90 \%$ (vol/vol) modified M199, 10 ng/mL of EGF (mouse EGF; Sigma), 10 IU/mL of hCG, 10 IU/mL of pregnant mare's serum gonadotropin (PMSG), $0.57 \mathrm{mM}$ cysteine (Sigma), and 10\% (vol/vol) pig follicular fluid, and then covered with $200 \mu \mathrm{L}$ paraffin oil in a four-well dish (NUNC) for $26 \mathrm{~h}$ [for COCs at metaphase I(MI)] or $44 \mathrm{~h}$ [for COCs at metaphase II (MII)] at $38.58^{\circ} \mathrm{C}$ in a $5 \% \mathrm{CO}_{2}$ atmosphere.

\section{LIMK1/2 inhibitor LIMKi 3 treatment}

For LIMK1/2 inhibitor treatment, stock LIMKi 3 (50 mM in dimethylsulfoxide [DMSO]) was 
233 diluted in M199 to final concentrations of 100 and $200 \mu \mathrm{M}$. A control group was cultured in DMSO

234 at the same relative concentration of solvent. COCs were cultured with LIMKi 3 to evaluate its

235 effects on oocyte maturation. COCs were denuded of their cumulus cells by gentle pipetting with

$2360.1 \%(\mathrm{w} / \mathrm{v})$ hyaluronidase (Sigma). Oocytes with clearly extruded polar bodies were considered to

237 be matured. The occurrence of first polar body extrusion in oocytes was examined after removing

238 cumulus cells.

239

240

241

242

243

244

245

246

247

248

249

250

251

252

253

\section{Rescue experiment}

After cultured in TCM 199 maturation medium containing $200 \mu$ M LIMK inhibitor LIMKi 3

for $44 \mathrm{~h}$, treated oocytes were washed three times in fresh culture solution ( 2 min each wash).

Oocytes were then transferred to fresh culture solution and cultured for an additional $16 \mathrm{~h}$ under paraffin oil at $38.5{ }^{\circ} \mathrm{C}$ in a $5 \% \mathrm{CO}_{2}$ atmosphere.

\section{Immunofluorescence staining and confocal microscopy}

For immunofluorescence staining of LIMK1/2, actin, and $\alpha$-tubulin, oocytes were fixed in 4\% paraformaldehyde in PBS at room temperature for $30 \mathrm{~min}$ and then transferred to a membrane permeabilization solution $(0.5 \%$ Triton $\mathrm{X}-100)$ for $8-12 \mathrm{~h}$ at room temperature. To suppress nonspecific binding of IgG, oocytes were blocked in blocking buffer for $1 \mathrm{~h}$ at room temperature (1\% BSA-supplemented PBS). Then oocytes were incubated overnight at $4^{\circ} \mathrm{C}$ or 5 hours at room temperature with a rabbit polyclonal p-LIMK-1/2 antibody(1:50) or anti- $\alpha$-tubulin-FITC(1:200) for 3 hours or with $2 \mu \mathrm{g} / \mathrm{mL}$ of Phalloidin-TRITC at room temperature for 1 hour. After three washes $(2 \mathrm{~min}$ each) in wash buffer $(0.1 \%$ Tween 20 and $0.01 \%$ Triton X-100 in PBS), oocytes were labeled with Alexa Fluor-488 goat anti-rabbit IgG (1:100; for p-LIMK-1/2 staining) for $1 \mathrm{~h}$ 
254 at room temperature. Oocytes were stained with Hoechst 33342 for 10 min, mounted on glass

255 slides, and then examined with a confocal laser-scanning microscope (Zeiss LSM 700 META,

256 Oberkochen, Germany). Each experiment was repeated three times; at least 30 oocytes were

257 examined per experimental condition.

258 Fluorescence intensity analysis

259 To analyze the fluorescence intensity of actin filaments, samples of control and treated 260 oocytes were mounted on the same glass slide. Image $\mathrm{J}$ software was used to define a region of 261 interest (ROI), and the average fluorescence intensity per unit area within the ROI was determined.

262 Independent measurements using identically sized ROIs were taken for the cell membrane and 263 cytoplasm. The average values of all measurements were used to determine the final average 264 intensities between control and treated oocytes.

265 Western Blot Analysis

266 A total of 100 porcine oocytes were collected, lysed in Laemmli sample buffer (SDS sample

267 buffer with 2-mercaptoethanol), and boiled at $100^{\circ} \mathrm{C}$ for $10 \mathrm{~min}$. Proteins were separated by SDS-

268 PAGE and then electrophoretically transferred to polyvinylidene fluoride membranes. To avoid 269 nonspecific binding, membranes were blocked with Tris-buffered saline (TBS) containing $0.1 \%$ $270(\mathrm{w} / \mathrm{w})$ Tween $20(\mathrm{TBST})$ and 5\% (w/v) nonfat dry milk powder for $1 \mathrm{~h}$ at room temperature. The 271 membranes were simultaneously incubated at overnight $4^{\circ} \mathrm{C}$ with a rabbit polyclonal p-LIMK-1/2 272 (1:200, Santa Cruz, USA) or rabbit monoclonal anti- $\alpha$-tubulin antibody (1:2000; Cell Signaling 273 Technology, Danvers, MA, USA). After washing three times in TBST (10 min each), the 274 membranes were incubated for $2 \mathrm{~h}$ with secondary anti-rabbit HRP-conjugated antibodies (1:2000; 
275 Cell Signaling Technology, Beverly, MA, USA) in 5\% nonfat dry milk in TBST at room

276

277

278

279

280

281

282

283

284

285

286

287

288

289

290

291

292

293

294

295

296

297

298

299

300

301

302

303

304

temperate. Finally, the membranes were washed 3 times in TBST and then the specific proteins

were visualized using chemiluminescence reagent (Millipore, Billerica, MA).

\section{Statistical Analysis}

A group of 80 oocytes were cultured with or without LIMKi 3 for once experimental condition and at least 3 replicates were performed for each treatment. Results were expressed as means \pm standard errors of the mean. Statistical analysis was conducted using analysis of variance (ANOVA), and differences between treatment groups were assessed by Duncan's multiple comparison test. $\mathrm{P}<0.05$ was considered significant.

\section{References}

Almonacid M, Terret ME, and Verlhac MH. 2014. Actin-based spindle positioning: new insights from female gametes. J Cell Sci 127:477-483.

Arber S, Barbayannis FA, Hanser H, Schneider C, Stanyon CA, Bernard O, and Caroni P. 1998. Regulation of actin dynamics through phosphorylation of cofilin by LIM-kinase. Nature 393:805-809.

Bamburg JR. 1999. Proteins of the ADF/cofilin family: essential regulators of actin dynamics. Annu Rev Cell Dev Biol 15:185-230.

Bernard O. 2007. Lim kinases, regulators of actin dynamics. Int J Biochem Cell Biol 39:1071-1076.

Brunet S, and Verlhac MH. 2011. Positioning to get out of meiosis: the asymmetry of division. Hum Reprod Update 17:68-75.

Dehapiot B, Carriere V, Carroll J, and Halet G. 2013. Polarized Cdc42 activation promotes polar body protrusion and asymmetric division in mouse oocytes. Dev Biol 377:202-212.

Deng M, Kishikawa H, Yanagimachi R, Kopf GS, Schultz RM, and Williams CJ. 2003. Chromatin-mediated cortical granule redistribution is responsible for the formation of the cortical granule-free domain in mouse eggs. Dev Biol 257:166-176.

Duan X, Liu J, Dai XX, Liu HL, Cui XS, Kim NH, Wang ZB, Wang Q, and Sun SC. 2014. Rho-GTPase effector ROCK phosphorylates cofilin in actin-meditated cytokinesis during mouse oocyte meiosis. Biol Reprod 90:37.

Gilchrist RB, Lane M, and Thompson JG. 2008. Oocyte-secreted factors: regulators of cumulus cell function and oocyte quality. Hum Reprod Update 14:159-177.

Halet G, and Carroll J. 2007. Rac activity is polarized and regulates meiotic spindle stability and anchoring in 
mammalian oocytes. Dev Cell 12:309-317.

Kaji N, Muramoto A, and Mizuno K. 2008. LIM kinase-mediated cofilin phosphorylation during mitosis is required for precise spindle positioning. J Biol Chem 283:4983-4992.

Li X, Zhu Y, Cao Y, Wang Q, Du J, Tian J, Liang Y, and Ma W. 2016. LIM kinase activity is required for microtubule organising centre positioning in mouse oocyte meiosis. Reprod Fertil Dev.

Liu XJ. 2012. Polar body emission. Cytoskeleton (Hoboken) 69:670-685.

Ma C, Benink HA, Cheng D, Montplaisir V, Wang L, Xi Y, Zheng PP, Bement WM, and Liu XJ. 2006. Cdc42 activation couples spindle positioning to first polar body formation in oocyte maturation. Curr Biol 16:214-220.

Maddox AS, Azoury J, and Dumont J. 2012. Polar body cytokinesis. Cytoskeleton (Hoboken) 69:855-868.

Maekawa M, Ishizaki T, Boku S, Watanabe N, Fujita A, Iwamatsu A, Obinata T, Ohashi K, Mizuno K, and Narumiya S. 1999. Signaling from Rho to the actin cytoskeleton through protein kinases ROCK and LIM-kinase. Science 285:895-898.

Maro B, and Verlhac MH. 2002. Polar body formation: new rules for asymmetric divisions. Nat Cell Biol 4:E281-283.

Ohashi K, Toshima J, Tajinda K, Nakamura T, and Mizuno K. 1994. Molecular cloning of a chicken lung cDNA encoding a novel protein kinase with N-terminal two LIM/double zinc finger motifs. J Biochem 116:636-642.

Okano I, Hiraoka J, Otera H, Nunoue K, Ohashi K, Iwashita S, Hirai M, and Mizuno K. 1995. Identification and characterization of a novel family of serine/threonine kinases containing two N-terminal LIM motifs. J Biol Chem 270:31321-31330.

Stanyon CA, and Bernard O. 1999. LIM-kinase1. Int J Biochem Cell Biol 31:389-394.

Sumi T, Matsumoto K, Takai Y, and Nakamura T. 1999. Cofilin phosphorylation and actin cytoskeletal dynamics regulated by rho- and Cdc42-activated LIM-kinase 2. J Cell Biol 147:1519-1532.

Sun SC, and Kim NH. 2013. Molecular mechanisms of asymmetric division in oocytes. Microsc Microanal 19:883-897.

Sutton-McDowall ML, Gilchrist RB, and Thompson JG. 2010. The pivotal role of glucose metabolism in determining oocyte developmental competence. Reproduction 139:685-695.

Takahashi T, Koshimizu U, Abe H, Obinata T, and Nakamura T. 2001. Functional involvement of Xenopus LIM kinases in progression of oocyte maturation. Dev Biol 229:554-567.

Yang N, Higuchi O, Ohashi K, Nagata K, Wada A, Kangawa K, Nishida E, and Mizuno K. 1998. Cofilin phosphorylation by LIM-kinase 1 and its role in Rac-mediated actin reorganization. Nature 393:809-812.

Yi K, and Li R. 2012. Actin cytoskeleton in cell polarity and asymmetric division during mouse oocyte maturation. Cytoskeleton (Hoboken) 69:727-737.

Zhang Y, Duan X, Cao R, Liu HL, Cui XS, Kim NH, Rui R, and Sun SC. 2014a. Small GTPase RhoA regulates cytoskeleton dynamics during porcine oocyte maturation and early embryo development. Cell Cycle 13:3390-3403.

Zhang Y, Duan X, Xiong B, Cui XS, Kim NH, Rui R, and Sun SC. 2014b. ROCK inhibitor Y-27632 prevents porcine oocyte maturation. Theriogenology 82:49-56.

\section{Figure legends}

Figure 1: p-LIMK-1/2 expression in porcine oocytes. (A) Subcellular localization of p-LIMK$1 / 2$ during oocyte meiosis in porcine. Double staining of $p$-LIMK-1/2 and actin, we found that $p$ - 
343 LIMK-1/2 accumulated at the cortex of oocytes from GV to the MII stage, which was co-localized

344 with actin. Green, p-LIMK-1/2; red, actin; blue, chromatin. Bar $=30 \mu \mathrm{m}$. (B) The protein 345 expression of p-LIMK-1/2 in porcine oocyte. Using western blot, the result showed that p-LIMK-

$3461 / 2$ was all expressed at the MI and MII stages.

347 Figure 2: LIMKi 3 treatment suppresses porcine oocyte maturation in vitro (A) LIMKi 3 treatment 348 efficiently restrained the diffusion of COCs. For COCs, cumulus cell expansion was weak after treatment with LIMKi 3; with significantly decreased diffusion rate of COCs $(p<0.05)$. Bar $=100$ $\mu \mathrm{m}$ (B) LIMKi 3 treatment affects the polar body excrusion of oocyte in porcine. Most oocytes of control were able to extrude polar body, whereas few oocytes extruded polar body after LIMKi 3 treatment and the rate of polar body extrusion decreased in a dose-dependent manner. Contrast to control group, the ratio of polar body extrusion was significantly lower with LIMKi 3 treatment at $100 \mu \mathrm{M}(\mathrm{p}<0.05), 150 \mu \mathrm{M}(\mathrm{p}<0.05)$ and $200 \mu \mathrm{M}(\mathrm{P}<0.01)$. Bar $=100 \mu \mathrm{m}(\mathrm{C})$ Compared with treated oocytes in $44 \mathrm{~h}$, after prolonging the culture time the maturation rate increased $(70.68 \% \pm$

(D) After rescuing oocytes from LIMKi 3, the maturation rate of

porcine oocyte increased.

Figure 3: LIMKi 3 treatment causes disruption of actin distribution in porcine oocyte (A) Immunofluorescence staining of actin distribution in oocyte membrane and cytoplasm after LIMKi

3 treatment. Oocytes that were treated with $200 \mu \mathrm{M}$ LIMKi 3 had obviously disturbed actin distribution. Both cortical actin and cytoplasmic actin were not visible in treated oocytes. Bar $=30$

$\mu \mathrm{m}$ (B) Average actin fluorescence intensity of MI oocytes. Actin fluorescence intensity was significantly lower than controls in LIMKi 3-treated oocytes at the membrane $(\mathrm{P}<0.01)$ and in 
364 the cytoplasm $(\mathrm{P}<0.01)$.

365 Figure 4: LIMKi 3 treatment causes abnormity of spindle positioning in porcine oocyte (A) The 366 positioning of spindle in oocyte. For controls, most spindles were located peripherally, while 367 spindles of LIMKi 3 treated oocytes were nearly centrally located. Enlarged images below show 368 the distances of spindles from the cortex. Bar $=30 \mu \mathrm{m}$ (B) Spindle stages and positioning after 26 $369 \mathrm{~h}$ in culture. Contrast with controls, the percentage of LIMKi 3 treated oocytes with peripherally 370 located spindles was significant lower $(\mathrm{p}<0.01)$. 
Figure 1 (on next page)

p-LIMK-1/2 expression in porcine oocytes

(A) Subcellular localization of $\mathrm{p}$-LIMK-1/2 during porcine oocyte meiotic maturation. Double staining of $\mathrm{p}-$ LIMK-1/2 and actin, we found that P-LIMK-1/2 accumulated at the cortex of oocytes from GV to the MII stage, which was co-localized with actin. Green, p-LIMK-1/2; red, actin; blue, chromatin. Bar $=30 \mu \mathrm{m}$. (B) The protein expression of $\mathrm{p}$-LIMK-1/2 in porcine oocyte. Using western blot, the result showed that $\mathrm{p}$-LIMK-1/2 was all expressed at the MI and MIl stages. 


\section{PeerJ}

Manuscript to be reviewed

A

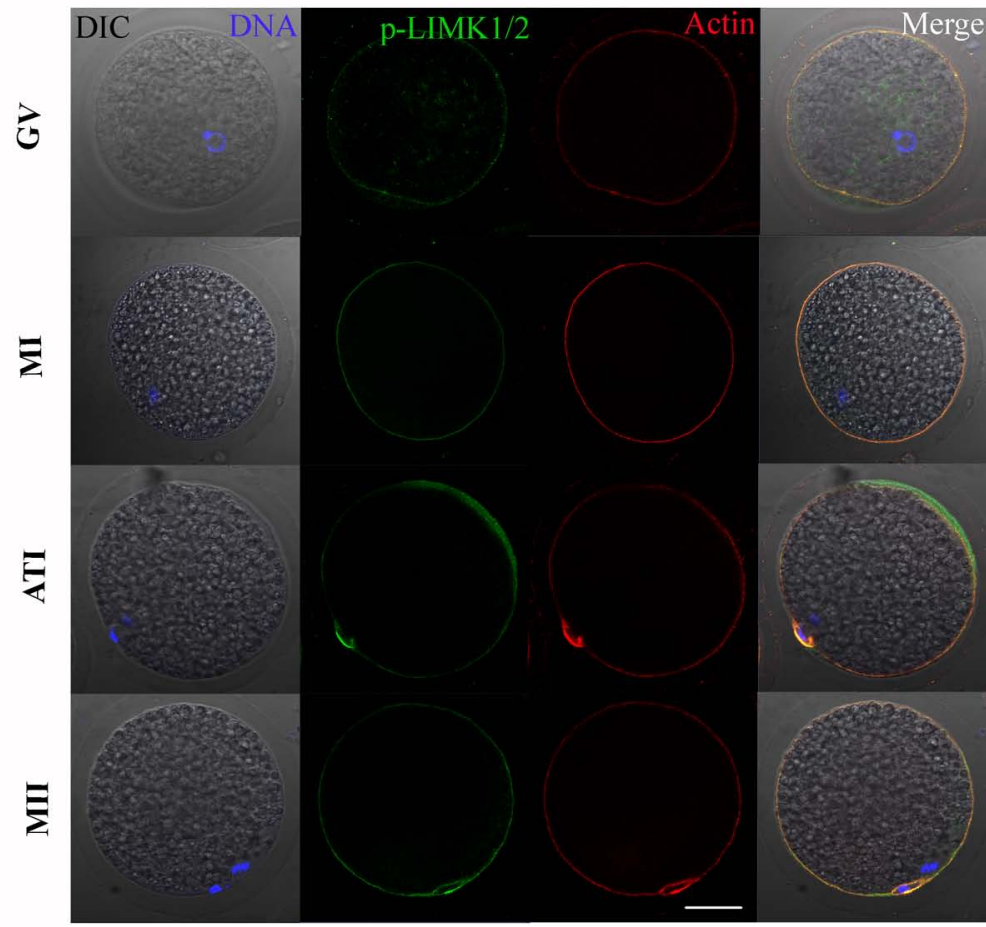

B

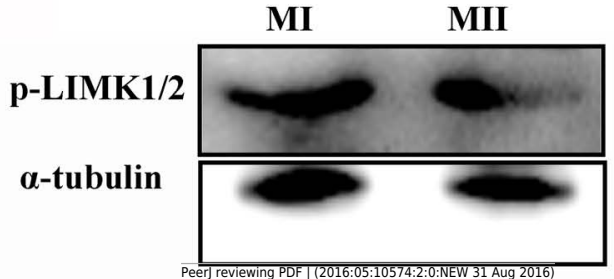




\section{Figure 2 (on next page)}

Figure 2

Figure 2: LIMKi 3 treatment suppresses porcine oocyte maturation in vitro (A) LIMKi 3 treatment efficiently restrained the diffusion of COCs. For COCs, cumulus cell expansion was weak after treatment with LIMKi 3; with significantly decreased diffusion rate of COCS $(p<0.05)$. Bar $=100 \mu \mathrm{m}$ (B) LIMKi 3 treatment affects the polar body excrusion of oocyte in porcine. Most oocytes of control were able to extrude polar body, whereas few oocytes extruded polar body after LIMKi 3 treatment and the rate of polar body extrusion decreased in a dose-dependent manner. Contrast to control group, the ratio of polar body extrusion was significantly lower with LIMKi 3 treatment at $100 \mu \mathrm{M} \square \mathrm{p}<0.05 \square, 150 \mu \mathrm{M}(\mathrm{p}<0.05)$ and $200 \mu \mathrm{M}($ $\mathrm{P}<0.01$ ). Bar $=100 \mu \mathrm{m}$ (C) Compared with treated oocytes in $44 \mathrm{~h}$, after prolonging the culture time the maturation rate increased $(70.68 \% \pm 6.99 \%$ vs. $47.61 \% \pm 7.92 \%)$. (D) After rescuing oocytes from LIMKi 3 , the maturation rate of porcine oocyte increased. 

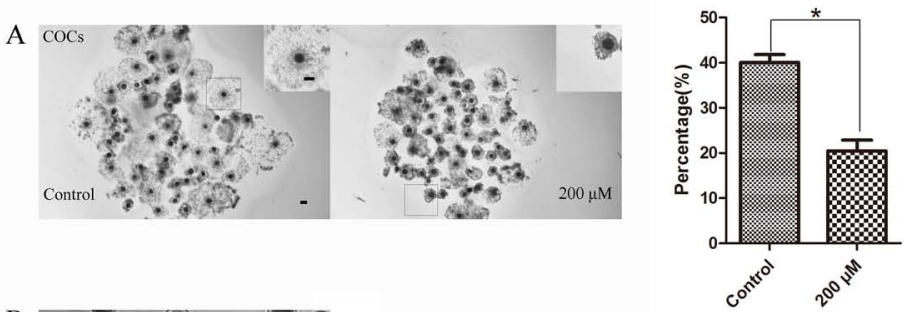

B
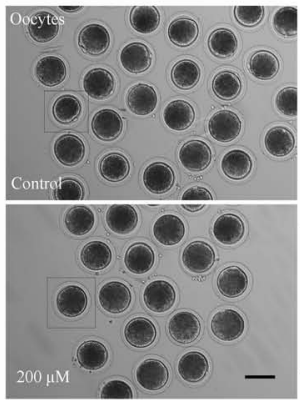

C

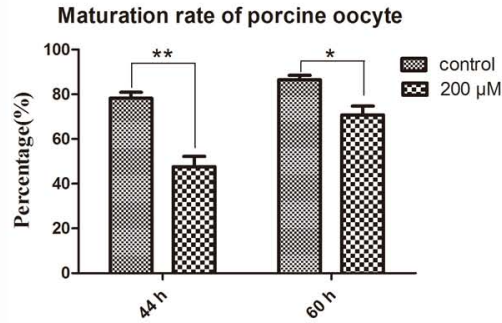

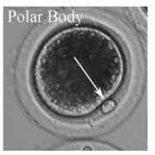

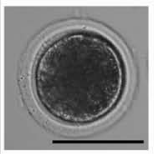

D

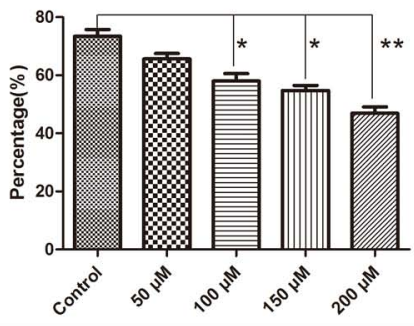

Rescue

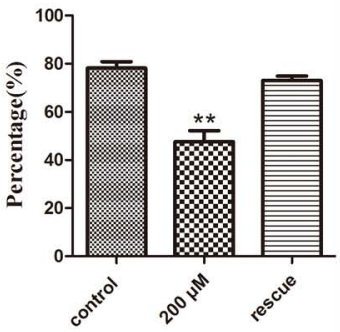


Figure 3 (on next page)

LIMKi 3 treatment causes disruption of actin distribution in porcine oocyte

(A) Actin distribution of Ml oocytes was examined by immunofluorescent staining. Oocytes that were treated with $200 \mu \mathrm{M}$ LIMKi 3 had profoundly disterbed actin distribution. Both cortical actin and cytoplasmic actin were not visible in treated oocytes. Bar $=30 \mu \mathrm{m}(\mathrm{B})$ Average actin fluorescence intensity of Ml oocytes. Actin fluorescence intensity was significantly lower in LIMKi 3-treated oocytes at the membrane $(P<0.01)$ and in the cytoplasm $(P<0.01)$. 


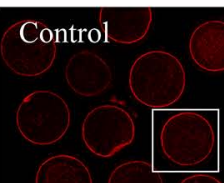

LIMKi 3

10X

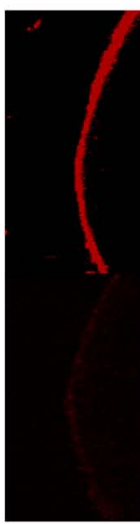

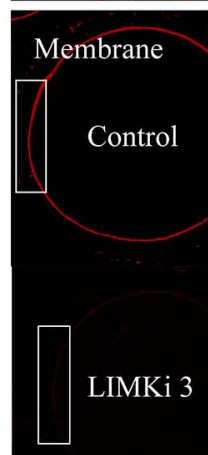

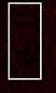

\section{B}

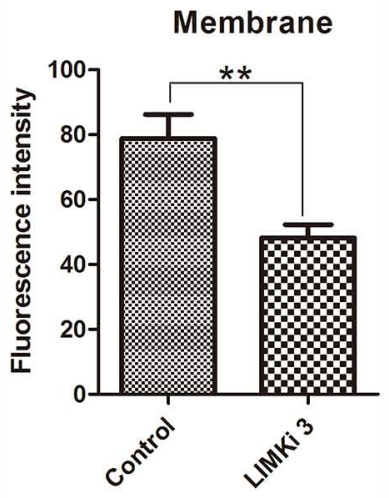


Figure 4 (on next page)

LIMKi 3 treatment causes abnormity of spindle position in porcine oocyte

(A) Spindle localization in oocyte. For controls, spindles were located peripherally, whereas they were nearly centrally located in LIMKi 3 treated oocytes. Enlarged images below show the distances of spindles from the cortex. Bar $=30 \mu \mathrm{m}$ (B) Spindle stages and positioning after $26 \mathrm{~h}$ in culture. Contrast with controls, the number of LIMKi 3 treated-oocytes with peripherally located spindles was significant lower $(p<0.01)$. 


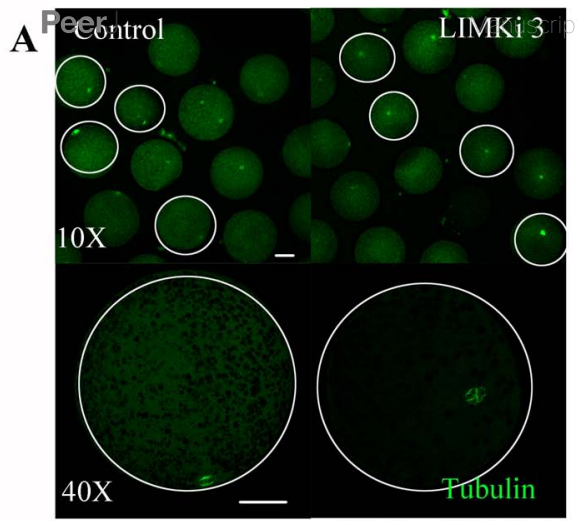

B

\section{Spindle positioning}

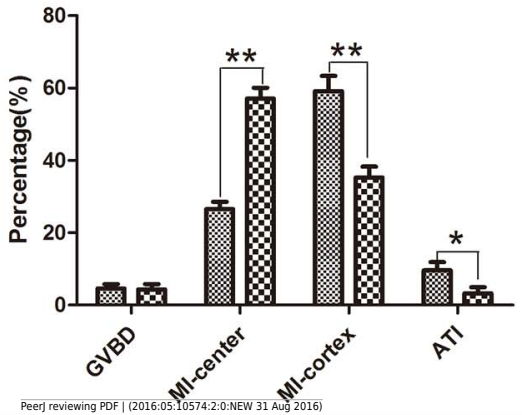

Control LIMKi 3 